University of Wollongong

Research Online

Faculty of Engineering and Information

Faculty of Engineering and Information

Sciences - Papers: Part B

Sciences

2015

Robust position control of a novel series elastic actuator via disturbance observer

Emre Sariyildiz

University of Wollongong, emre@uow.edu.au

Gong Chen

National Univeristy of Singapore

Haoyong Yu

National Univeristy of Singapore

Follow this and additional works at: https://ro.uow.edu.au/eispapers1

Part of the Engineering Commons, and the Science and Technology Studies Commons

Research Online is the open access institutional repository for the University of Wollongong. For further information contact the UOW Library: research-pubs@uow.edu.au 


\title{
Robust position control of a novel series elastic actuator via disturbance observer
}

\begin{abstract}
This paper proposes a new robust position control method for a novel series elastic actuator (SEA). It is a wellknown fact that SEAs provide many benefits in force control, e.g., lower reflected inertia and impedance, greater shock tolerance, safety, and so on. However, current SEA designs have a common performance limitation due to the compromise on the selection of spring stiffness. The performance of an SEA can be significantly improved by changing the stiffness of the spring; however, designing a variable-stiffness SEA is a quite challenging task. In this paper, a novel variable-stiffness SEA, which can relax the fundamental performance limitation of conventional SEAs, is proposed. It consists of torsional and linear springs, which have different compliances, in series. The soft and hard springs improve the performance when low and high force control applications are performed, respectively. Although SEAs have several advantages in force control, their position control problem is more complicated than the force control one. Moreover, using extra springs increases the number of vibration mode, which may significantly deteriorate the performance, in the position control problem of the SEA. In this paper, a new position control system, which improves the performance by increasing the robustness and suppressing the vibration, is proposed for a novel SEA. Experimental results are given to validate the proposal.
\end{abstract}

\section{Keywords}

via, disturbance, actuator, elastic, series, novel, control, position, robust, observer

\section{Disciplines}

Engineering | Science and Technology Studies

\section{Publication Details}

Sariyildiz, E., Chen, G. \& Yu, H. (2015). Robust position control of a novel series elastic actuator via disturbance observer. 2015 IEEE/RSJ International Conference on Intelligent Robots and Systems (IROS) (pp. 5423-5428). United States: IEEE. 


\title{
Robust Position Control of a Novel Series Elastic Actuator via Disturbance Observer
}

\author{
Emre Sariyildiz, Gong Chen, Haoyong Yu, Member, IEEE
}

\begin{abstract}
This paper proposes a new robust position control method for a novel series elastic actuator (SEA). It is a wellknown fact that SEAs provide many benefits in force control, e.g., lower reflected inertia and impedance, greater shock tolerance, safety, and so on. However, current SEA designs have a common performance limitation due to the compromise on the selection of spring stiffness. The performance of an SEA can be significantly improved by changing the stiffness of the spring; however, designing a variable-stiffness SEA is a quite challenging task. In this paper, a novel variable-stiffness SEA, which can relax the fundamental performance limitation of conventional SEAs, is proposed. It consists of torsional and linear springs, which have different compliances, in series. The soft and hard springs improve the performance when low and high force control applications are performed, respectively. Although SEAs have several advantages in force control, their position control problem is more complicated than the force control one. Moreover, using extra springs increases the number of vibration mode, which may significantly deteriorate the performance, in the position control problem of the SEA. In this paper, a new position control system, which improves the performance by increasing the robustness and suppressing the vibration, is proposed for a novel SEA. Experimental results are given to validate the proposal.
\end{abstract}

\section{INTRODUCTION}

Stiff and non-back-drivable actuators have been widely used in the conventional applications of robots, e.g., industrial robot tasks that require high position accuracy and repeatability [1-3]. Although they can intrinsically provide high performance position control systems thanks to the robust mechanical structures, they mainly suffer from performing fine motion tasks such as grinding and polishing industrial applications, human-robot interaction, medical robot implementations, and so on [4 and 5]. Insufficient force control performance and safety are one of the main challenging issues of the conventional stiff and non-backdrivable actuators [1 and 2].

In the last two decades, the demands on the compliant robots have been rapidly increased to perform more dexterous and versatile tasks, i.e., fine motions [1, 6 and 7]. Series elastic actuators (SEA) can intrinsically improve the performance of such tasks, i.e., force control, since they

This work was supported by the Seed Fund of the Engineering Design and Innovation Center, National University of Singapore,Singapore under Grant no. R-261-503-002-133.

E. Sariyildiz, G. Chen and H. Yu are with the Department of Biomedical Engineering, Faculty of Engineering, National University of Singapore, 9 Engineering Drive 1, Singapore 117576 (emails: bieemre@nus.edu.sg, chen.gong@u.nus.edu, bieyhy@nus.edu.sg ) have several benefits such as lower reflected inertia, low cost force measurement, safety, and so on [8 and 9]. Although it is basically a compliant actuator, SEA is generally used instead of compliancy to emphasize the passive compliant structure. Current SEA designs have a common problem of performance limitation due to the compromise on the selection of spring stiffness [10]. Besides, although SEAs have several advantages in force control, their position control problem is harder than the conventional stiff actuators' one. Therefore, more advanced position control systems should be designed to improve the position control performance of SEAs.

In this paper, a new robust position control method is proposed for a novel SEA by using resonance ratio control (RRC) method [11]. The proposed SEA is designed by using two springs in series: a torsional hard spring and a linear soft spring. The soft and hard springs are proposed to improve the performances of low and high force control applications, respectively. In other words, a simple variable-stiffness SEA is proposed to overcome the fundamental performance limitation problem. However, the motion control problem of the proposed novel SEA is more complicated than the conventional SEAs' one. Using extra spring increases the number of vibration mode, which significantly deteriorates the performance, in position control. To improve the performance of the proposed SEA, a new robust position control method is proposed. Disturbance observer (DOb) is used to achieve high performance robust acceleration-based position control system. However, the robustness deteriorates the performance of position control by increasing the vibration at tip point. The vibration is simply suppressed by adjusting the ratio between resonant and antiresonant frequencies, i.e., using RRC [11 and 12]. In RRC, the robustness deteriorates by feeding-back torsional disturbances to suppress vibration. However, servo system becomes more sensitive to external disturbances since the robustness deteriorates. To suppress the external disturbances, i.e., improve the robustness, an Arm DOb is used. The proposed method can be directly applied to the conventional SEAs to improve their performances.

The rest of the paper is organized as follows. In section II, the novel SEA is proposed. In section III, DOb-based robust motion control systems are briefly explained. In section IV, a new robust position controller is proposed for the novel SEA. In section V, the validity of the proposal is verified by giving experimental results. The paper ends with conclusion given in the last section. 


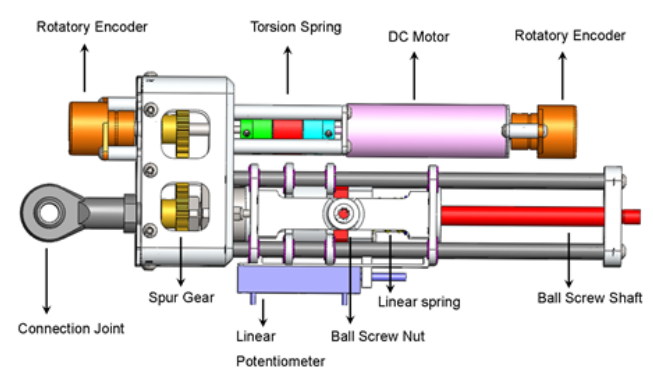

(a) Principle of the actuator design (CAD model).

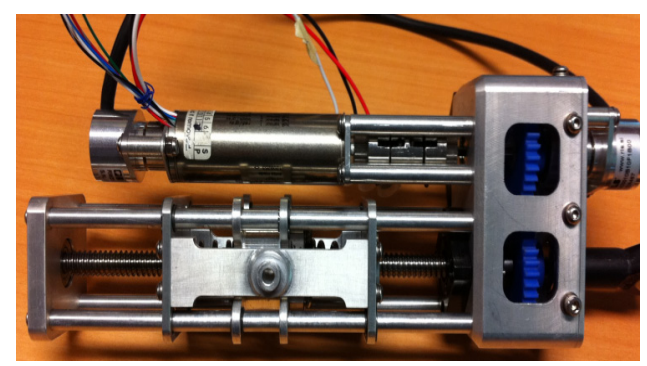

(b) A prototype of the novel compliant actuator.

Fig.1. Proposed novel SEA.

\section{Novel Series Elastic Actuator}

In this paper, a novel variable-stiffness SEA, which is illustrated in Fig. 1a, is proposed. It consists of a rotary servomotor with a rotary encoder, a torsional spring assembly with another rotary encoder, a pair of spur gear with appropriate gear ratio to transmit the motion to the ball screw which converts the rotary motion of the shaft to linear motion of the nut, a set of linear springs attached to the ball screw nut to transmit the force to a carriage which has an force output pin to transmit the force to the load (prospective robot link), and a linear position sensor installed in the carriage to measure the displacement of the linear spring. The angular deflection of the torsional spring is estimated by using the rotary encoders, and the deflection of the linear spring is estimated by using rotary and linear encoders. The first prototype of the proposed novel SEA is shown in Fig. $1 \mathrm{~b}$.

In this design, the variable stiffness is achieved by using torsional and linear springs which have different compliancy. The linear spring is chosen to provide average targeted torque, i.e., small torque; therefore, it is soft, small and light-weight. As the stiffness of the linear spring is decreased, safety of fine motions is improved; however, the performance deteriorates due to the bandwidth limitation, i.e., fine motions can be performed slowly. A very small torsional spring is used to achieve a very big effective spring constant at the output end because it is located in the high speed range. Fig. 2a illustrates the dynamic model of the novel SEA. Due to the difference in spring constant, when the actuator is working in the low force range, the force control is based on the linear spring and the torsional spring behaves like a rigid link as shown in Fig. 2b. However, when the actuator is working in the high force range, the soft linear spring is fully compressed and the force control is based on the torsional spring as shown in Fig. 2c.

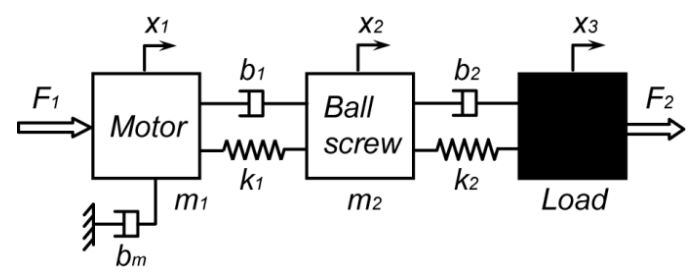

(a) General case.

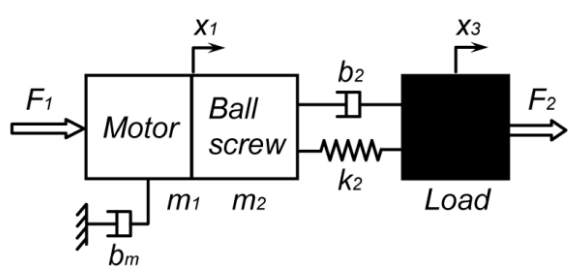

(b) Model for low force range.

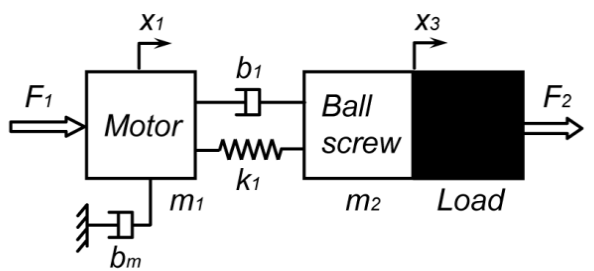

(c) Model for high force range.

Fig.2. Modeling of the novel SEA in translational motion.

It is obvious that the proposed novel SEA can provide several advantages, such as safety, in force control. However, its position control problem is more complicated than conventional stiff actuators' one. Therefore, more advanced position controllers should be designed.

\section{Robust Motion CONTROL Via DOB}

Block diagram of a DOb is shown in Fig. 3 [13]. In this figure:

$J_{m}, J_{m n} \quad$ Actual and nominal inertias;

$K_{\tau}, K_{\tau n} \quad$ Actual and nominal torque coefficients;

$I^{m} \quad$ Motor current;

$\tau_{\text {dis }}, \hat{\tau}_{\text {dis }} \quad$ Total disturbance and its estimation;

$g_{D O b} \quad$ Cut-off frequency of DOb;

$\tau_{d} \quad$ External disturbance including friction, load and interactive forces;

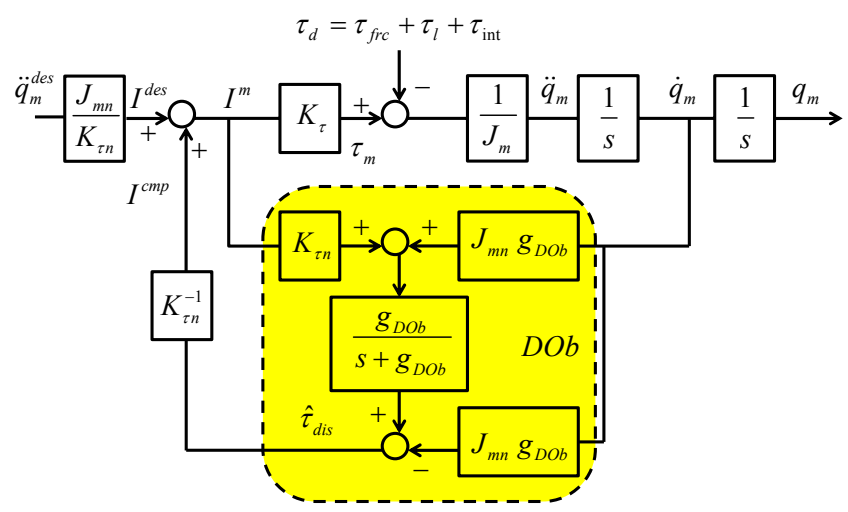

Fig.3. Block diagram of DOb. 
$q_{m}, \dot{q}_{m}, \ddot{q}_{m} \quad$ Angle/position, velocity and acceleration; $\ddot{q}_{m}^{\text {des }} \quad$ Desired acceleration.

$\mathrm{A} \mathrm{DOb}$ is essential to design high performance acceleration-based robust motion control systems, i.e. position and force control systems [13 and 14]. It estimates external disturbances and system uncertainties such as inertia variation, friction, external load, and so on [15 and 16]. The output of DOb, which is estimated disturbance, is fed-back so that the robustness of a motion control system is simply achieved as shown in Fig. 3. The bandwidth of DOb $g_{D O b}$ is directly related to the robustness of the motion control system, and the higher the bandwidth of DOb is, the more the robustness improves. However, it is limited by practical constraints such as noise and sampling time. Practical analysis and design methods for DOb-based motion control systems can be found in [13 and 14].

Although, in general, the robustness improves the performance of motion control systems by suppressing disturbances, it degrades the performance of SEAs by increasing vibration. Therefore, conventional robust control systems cannot be directly applied to the position control problem of SEA. An advanced robust motion controller is proposed for the novel SEA in the next section.

\section{Robust Position CONTROL OF Novel SEA}

Block diagram of the two-mass resonant system, which is illustrated in Fig. 2b and Fig. 2c, is shown in Fig. 4a when damping force is neglected, i.e., $b_{\bullet}=0$. If it is assumed that $K_{\Gamma}=m_{1}^{-1}$, then Fig. $4 \mathrm{~b}$ can be directly obtained without any approximation for the two-mass resonant system. In this figure, $K_{\Gamma}$ denotes the feed-back coefficient of reaction force and is used to adjust the resonance ratio of the compliant actuator. The transfer functions of the two-mass resonant system are directly derived from Fig. $4 \mathrm{~b}$ as follows:

$$
\begin{gathered}
x_{\bullet}=\frac{\frac{k_{\bullet}}{m_{\bullet}}}{s^{2}+\frac{k_{\bullet}}{m_{\bullet}}} x_{1}=\frac{w_{a}^{2}}{s^{2}+w_{a}^{2}} x_{1} \\
x_{1}=\frac{s^{2}+\frac{k_{\bullet}}{m_{\bullet}}}{s^{2}+\frac{k_{\bullet}}{m_{\bullet}}\left(1+K_{\Gamma} m\right)} \frac{1}{s^{2}} \ddot{x}_{1}^{d e s}=\frac{s^{2}+w_{a}^{2}}{s^{2}+w_{m}^{2}} \frac{1}{s^{2}} \ddot{x}_{1}^{\text {des }}
\end{gathered}
$$

where $k_{\bullet}=k_{2}, m_{\bullet}=m_{3}$ and $x_{\bullet}=x_{3}$ when Fig. $2 \mathrm{~b}$ is considered; and $k_{\bullet}=k_{1}, m_{\bullet}=m_{2}+m_{3}$ and $x_{\bullet}=x_{2}$ when Fig. $2 \mathrm{c}$ is considered.

The plant model can be easily derived by using Eq. (1) and Eq. (2) as follows:

$$
x_{\bullet}=G_{2 M}(s) \ddot{x}_{1}^{d e s}=\frac{w_{a}^{2}}{s^{2}+w_{m}^{2}} \frac{1}{s^{2}} \ddot{x}_{1}^{d e s}
$$

Here, $w_{a}$ is the pole/zero of the arm/motor portion's transfer function which is given in Eq.(1)/Eq. (2) and represents anti-resonant frequency in the motor portion; however, $w_{m}$ is the pole of the motor portion and represents

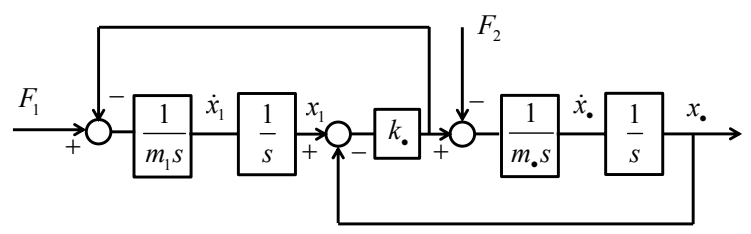

(a) Two-mass resonant system.

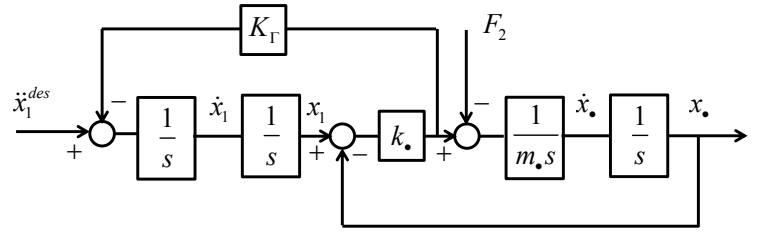

(b) Resonance ratio control of two-mass resonant system.

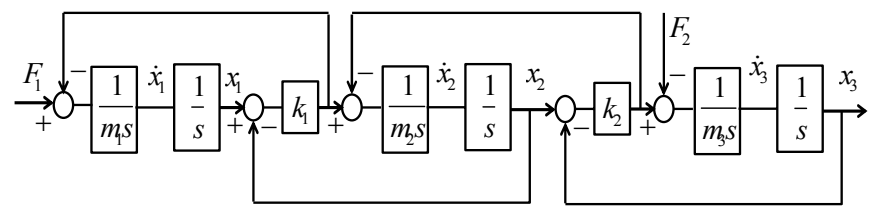

(c) Three-mass resonant system.

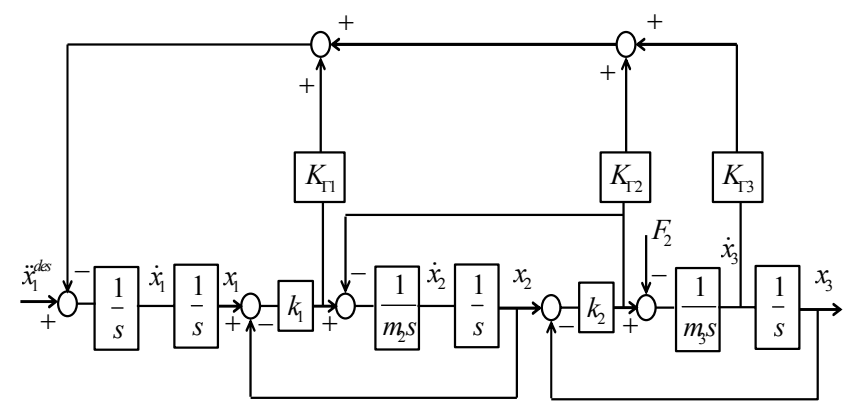

(d) Resonance ratio control of three-mass resonant system.

Fig.4. Block diagrams of resonant systems.

the resonant frequency. The ratio between the resonant and anti-resonant frequencies is derived by using

$$
K_{\text {Rratio }}=\frac{w_{m}}{w_{a}}=\frac{\sqrt{\frac{k_{\bullet}}{m_{\bullet}}\left(1+K_{\Gamma} m_{\bullet}\right)}}{\sqrt{\frac{k_{\bullet}}{m_{\bullet}}}}=\sqrt{1+K_{\Gamma} m_{\bullet}}
$$

In the conventional RRC method, it is shown that the vibration of compliant actuators can be suppressed by controlling the ratio between resonant and anti-resonant frequencies via reaction force feed-back $K_{\Gamma}$ [11].

Let us assume that the position controller is designed as shown in Fig. 5. The characteristic function of the RRC system is directly derived from Fig. 5 as follows:

$$
\Delta(s)=s^{4}+K_{v} s^{3}+\left(w_{m}^{2}+K_{p}\right) s^{2}+w_{a}^{2} K_{v} s+w_{a}^{2} K_{p}
$$

Let us also define the desired characteristic function by using

$$
\Delta(s)=\prod_{i=1}^{2}\left(s^{2}+2 \xi_{i} w_{i} s+w_{i}^{2}\right)
$$

The control parameters of the RRC system are simply derived by using pole placement method as follows: 


$$
K_{p}=w_{a}^{2}, \quad K_{v}=4 w_{a} \quad \text { and } \quad K_{\Gamma}=\frac{4}{m .}
$$

An Arm DOb is used to estimate external disturbances as shown in Fig. 5. The estimated disturbance is fed-back to achieve the robustness of the motion control system by using the transfer function between $x^{\text {ref }}$ and $F_{2}$.

$$
\frac{x^{r e f}}{F_{2}}=G_{F_{2}}^{r e f}(s)=-\frac{s^{2}+K_{v} s+K_{p}+k_{\bullet} K_{\Gamma}}{k_{\bullet} K_{p}}
$$

The conventional RRC can be used in the position control problem of the novel SEA if

- It is approximately modeled by neglecting the compliancy of hard spring

- Soft spring is fully compressed during position control

However, in reality, the dynamic response is influenced by the compliancy of two springs. Therefore, an advanced resonance ratio controller should be designed to suppress the vibration and improve the performance of the novel SEA. It can be similarly designed by using the conventional RRC method as follows:

The transfer functions of the three-mass resonant system are directly derived from Fig. $4 \mathrm{c}$ as follows:

Arm Portion

$$
x_{3}=\frac{\frac{k_{1}}{m_{2}} \frac{k_{2}}{m_{3}}}{s^{4}+\left(\frac{k_{1}+k_{2}}{m_{2}}+\frac{k_{2}}{m_{3}}\right) s^{2}+\frac{k_{1} k_{2}}{m_{2} m_{3}}} x_{1}=\frac{w_{a 1}^{2} w_{a 2}^{2}}{\left(s^{2}+w_{a 1}^{2}\right)\left(s^{2}+w_{a 2}^{2}\right)} x_{1}
$$

Motor Portion:

$$
\begin{aligned}
x_{2} & =\frac{s^{4}+\left(\frac{k_{1}+k_{2}}{m_{2}}+\frac{k_{2}}{m_{3}}\right) s^{2}+\frac{k_{1} k_{2}}{m_{2} m_{3}}}{s^{4}+\left(\frac{k_{1}+k_{2}}{m_{2}}+\frac{k_{2}}{m_{3}}+\frac{k_{1}}{m_{1}}\right) s^{2}+\frac{k_{1} k_{2}}{m_{1} m_{2} m_{3}}\left(m_{1}+m_{2}+m_{3}\right)} \frac{1}{s^{2}} \ddot{x}_{1}^{\text {des }} \\
& =\frac{\left(s^{2}+w_{a 1}^{2}\right)\left(s^{2}+w_{a 2}^{2}\right)}{\left(s^{2}+w_{m 1}^{2}\right)\left(s^{2}+w_{m 2}^{2}\right)} \frac{1}{s^{2}} \ddot{x}_{1}^{\text {des }}
\end{aligned}
$$

The plant model can be easily derived by using Eq. (9) and Eq. (10) as follows:

$$
x_{3}=G_{3 M}(s) \ddot{x}_{1}^{d e s}=\frac{w_{a 1}^{2} w_{a 2}^{2}}{\left(s^{2}+w_{m 1}^{2}\right)\left(s^{2}+w_{m 2}^{2}\right)} \frac{1}{s^{2}} \ddot{x}_{1}^{\text {des }}
$$

Here, $w_{a 1}$ and $w_{a 2}$ denote motor portion's anti-resonant frequencies and $w_{m 1}$ and $w_{m 2}$ denote motor portion's resonant frequencies. Since the order of the plant is six and a simple $P$ controller with velocity feed-back is used in the position control, three control parameters, which are shown in Fig. $4 \mathrm{~d}$, should be fed-back to suppress all vibration modes. If only two control parameters $K_{\Gamma 1}$ and $K_{\Gamma 2}$ are used, e.g. in [12], then the damping of the system cannot be precisely controlled; however, the vibration can be suppressed by applying extra damping which may degrade the performance.

Let us assume that $K_{\Gamma 3}=0$ which was proposed to suppress higher order vibration modes of a flexible

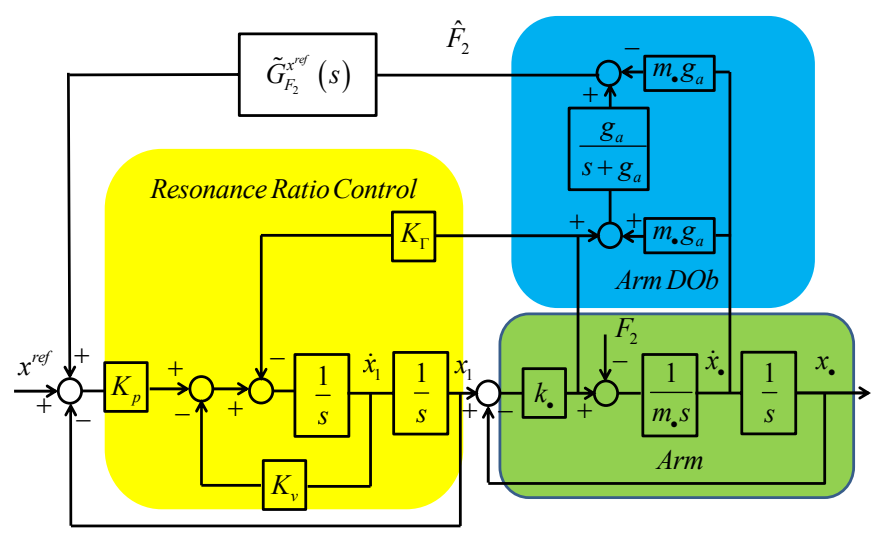

Fig.5. Position control of two-mass resonant system.

manipulator in [12]. The characteristic function of the closed loop system is derived from Fig. $4 \mathrm{~d}$ and Fig. 5 as follows:

$$
\begin{array}{r}
\Delta(s)=s^{6}+K_{v} s^{5}+\left(w_{m 1}^{2}+w_{m 2}^{2}+K_{p}\right) s^{4}+\left(w_{a 1}^{2}+w_{a 2}^{2}\right) K_{v} s^{3}+ \\
\left(\left(w_{a 1}^{2}+w_{a 2}^{2}\right) K_{p}+w_{m 1}^{2} w_{m 2}^{2}\right) s^{2}+w_{a 1}^{2} w_{a 2}^{2} K_{v} s+w_{a 1}^{2} w_{a 2}^{2} K_{p}
\end{array}
$$

where

$$
\begin{gathered}
w_{a 1}^{2}+w_{a 2}^{2}=\frac{k_{1}+k_{2}}{m_{2}}+\frac{k_{2}}{m_{3}} \\
w_{a 1}^{2} w_{a 2}^{2}=\frac{k_{1} k_{2}}{m_{2} m_{3}} \\
w_{m 1}^{2}+w_{m 2}^{2}=\frac{k_{1}+k_{2}}{m_{2}}+\frac{k_{2}}{m_{3}}+k_{1} K_{\Gamma 1} \\
w_{m 1}^{2} w_{m 2}^{2}=\frac{k_{1} k_{2}}{m_{2} m_{3}}+\frac{k_{1} k_{2}}{m_{2}}\left(K_{\Gamma 1}+K_{\Gamma 2}\right)+\frac{k_{1} k_{2}}{m_{3}} K_{\Gamma 1}
\end{gathered}
$$

Let us define the desired characteristic function by using

$$
\Delta(s)=\prod_{i=1}^{3}\left(s^{2}+2 \xi_{i} w_{i} s+w_{i}^{2}\right)
$$

Since there are four control parameters, $K_{p}, K_{v}, K_{\sqcap}$, and $K_{\Gamma 2}$, four poles of the characteristic function can be directly controlled. In other words, there are always two uncontrollable poles defined by $s^{2}+2 \xi_{\text {unc }} w_{u n c} s+w_{u n c}^{2}$ where $\xi_{\text {unc }}$ and $w_{u m c}$ represent uncontrollable damping coefficient and natural frequency.

Let us assume that $\xi_{1}=\xi_{2}=1$ to improve the stability and $w_{1}=w_{2}=w_{0}$ to achieve the fastest response [11 and 12]. The uncontrollable poles and control parameters are derived by using pole placement as follows:

$$
\begin{gathered}
w_{u m c}^{2}=\frac{w_{0}^{2}\left(\left(w_{a 1}^{2}+w_{a 2}^{2}\right) w_{0}^{2}-5 w_{a 1}^{2} w_{a 2}^{2}-w_{0}^{4}\right)}{\left(w_{a 1}^{2}+w_{a 2}^{2}\right) w_{0}^{2}-5 w_{0}^{4}-w_{a 1}^{2} w_{a 2}^{2}} \\
\xi_{\text {unc }}=2 \frac{w_{0}}{w_{u m c}} \frac{w_{0}^{2} w_{u m c}^{2}-w_{a 1}^{2} w_{a 2}^{2}}{w_{a 1}^{2} w_{a 2}^{2}-w_{0}^{4}} \\
K_{v}=4 w_{0}+2 w_{u m c} \xi_{u m c} \\
K_{p}=\frac{w_{0}^{4} w_{u m c}^{2}}{w_{a 1}^{2} w_{a 2}^{2}}
\end{gathered}
$$




$$
\begin{gathered}
K_{\Gamma 1}=\frac{6 w_{0}^{2}+w_{u m c}^{2}+8 w_{0} w_{u m c} \xi_{u m c}-K_{p}-w_{a 1}^{2}-w_{a 2}^{2}}{k_{1}} \\
K_{\Gamma 2}=\frac{w_{0}^{4}+6 w_{0}^{2} w_{u m c}^{2}+8 w_{0}^{3} w_{u m c} \xi_{u m c}-\left(w_{a 1}^{2}+w_{a 2}^{2}\right) K_{p}}{m_{3}\left(w_{a 1}^{2} w_{a 2}^{2}\right)}-\frac{\left(1+\left(m_{2}+m_{3}\right) K_{\Gamma 1}\right)}{m_{3}}
\end{gathered}
$$

It is clear from the equations that the performance of the position control system may drastically change by the uncontrollable poles, i.e. $\xi_{u m c}$ and $w_{u m c}$. Therefore, $K_{\Gamma 3}$ should be used to suppress all vibration modes of the novel SEA.

If $K_{\Gamma 3}$ is used, then the damping ratios of the all poles can be controlled by using pole placement. However, there is one uncontrollable natural frequency due to the insufficient control parameters $K_{p}, K_{v}, K_{\Gamma 1}, K_{\Gamma 2}$, and $K_{\Gamma 3}$.

Let us assume that $\xi_{1}=\xi_{2}=\xi_{3}=1$ to improve the stability and $w_{1}=w_{2}=w_{0}$ to achieve the fastest response $[11,12]$. The uncontrollable natural frequency and control parameters are derived by using pole placement as follows:

$$
w_{\text {uncl }, 2}=\frac{2\left(w_{a 1}^{2}+w_{a 2}^{2}\right)-12 w_{0}^{2} \pm \sqrt{\Psi}}{8 w_{0}}
$$

where $\Psi=\left(12 w_{0}^{2}-2\left(w_{a 1}^{2}+w_{a 2}^{2}\right)^{2}-64 w_{0}\left(w_{0}^{3}-\left(w_{a 1}^{2}+w_{a 2}^{2}\right) w_{0}\right)\right)$,

$$
\begin{gathered}
K_{v}=4 w_{0}+2 w_{u m c} \xi_{3} \\
K_{p}=\frac{w_{0}^{4} w_{u m c}^{2}}{w_{a 1}^{2} w_{a 2}^{2}} \\
K_{\Gamma 1}=\frac{6 w_{0}^{2}+w_{u m c}^{2}+8 w_{0} w_{u m c}-K_{p}-w_{a 1}^{2}-w_{a 2}^{2}}{k_{1}} \\
K_{\Gamma 2}=\frac{w_{0}^{4}+6 w_{0}^{2} w_{u m c}^{2}+8 w_{0}^{3} w_{u m c}-\left(w_{a 1}^{2}+w_{a 2}^{2}\right) K_{p}}{m_{3}\left(w_{a 1}^{2} w_{a 2}^{2}\right)}-\frac{\left(1+\left(m_{2}+m_{3}\right) K_{\Gamma 1}\right)}{m_{3}} \\
K_{\Gamma 3}=\frac{4 w_{0}^{3} w_{u m c}^{2}+2 w_{0}^{4} w_{u m c}}{w_{a 1}^{2} w_{a 2}^{2}}-K_{d}
\end{gathered}
$$

In the proposed RRC method, $w_{0}$ is used as a free design parameter. The higher $w_{0}$ is, the faster the system response is achieved. However, $w_{0}$ cannot be freely increased since the stability deteriorates.

External disturbances can be similarly suppressed by using an Arm DOb. In three-mass RRC system, the transfer function between $x^{\text {ref }}$ and $F_{2}$ is derived as follows:

$$
\frac{x^{\text {ref }}}{F_{2}}=\frac{\left(s^{2}+K_{v} s+K_{p}+k_{1} K_{\Gamma 1}\right)\left(m_{2} s^{2}+k_{1}+k_{2}\right)-k_{1}\left(k_{1} K_{\Gamma 1}-k_{2} K_{\Gamma 2}\right)}{k_{1} k_{2} K_{p}}
$$

\section{EXPERIMENTAL RESULTS}

In this section, robust position control experimental results of the novel SEA are given. Specifications of the experimental setup are shown in Table I, and the SEA that is used in experiments is shown in Fig. 1b. The controller is the NI CompactRIO 9074 embedded FPGA system with NI
TABLE I. SPECIFICATIONS OF THE EXPERIMENTAL SETUP

\begin{tabular}{|c|c|c|}
\hline Parameter & Value & Explanation \\
\hline$J_{m}$ & $8.9 \mathrm{gcm}^{2}$ & Motor Inertia \\
\hline$k_{1}$ & $0.29 \mathrm{Nm} / \mathrm{rad}$ & Spring Stiffness \\
\hline$k_{2}$ & $0.0024 \mathrm{Nm} / \mathrm{rad}$ & Spring Stiffness \\
\hline$J_{m 2}$ & $2.76 \times 10^{-6} \mathrm{kgm}^{2}$ & Gear and Screw Inertia \\
\hline$J_{m 3}$ & $0.0025 \mathrm{kgm}^{2}$ & Link Inertia \\
\hline
\end{tabular}

9263 (Analog Output module), and NI 9516 (Encoder module) for the data acquisition. The sampling frequency is $2 \mathrm{KHz}$.

Fig. 9 shows the position control responses of the SEA when a step reference input, which is $16 \mathrm{~mm} /$ 50rad., is applied at $1 \mathrm{~s}$., and a disturbance is applied at 5 s.. Fig. 9a shows the position control responses when a simple PD position control system is used. It is clear from the figure that high position control gains are required to improve the performance of the position control system. However, as it is shown in the figure, increasing the position control gain degrades the stability so the damping control parameter should be adjusted properly. Besides, it is a well-known fact that the control gain cannot be freely increased due to practical constraints such as exciting high frequency dynamics. The difference between motor and arm positions, i.e., $x_{1}$ and $x_{3}$, is due to non-linear friction disturbance. Fig. $9 \mathrm{~b}$ shows the position control responses when a DOb is used to improve the robustness of the system. It clearly indicates that the steady state performance of the SEA can be significantly improved by using DOb; however, although the transient response is improved at motor side, it significantly deteriorates due to vibration at arm side. Besides, the nonlinear friction cannot be removed at arm side, since DOb is designed in the motor side due to stability issues. Fig. 9c shows the position control responses when the proposed resonance ratio controller and friction compensation given in $[10,14]$ are used. The proposed controller can significantly improve the performance of the SEA by decreasing the robustness. However, the motion control system becomes sensitive to external disturbances as shown in the figure. The robustness of the system can be improved by using an Arm DOb. Fig. 9d shows that external disturbances can be suppressed and the performance of the SEA can be improved by using the proposed robust RRC system. Since the approximation of the transfer function between $x^{\text {ref }}$ and $F_{2}$ is used due to practical issues in the design of the control system, only constant disturbances can be eliminated.

\section{CONCLUSION}

This paper proposes a robust position control system for a novel SEA by using RRC. Although SEAs have significant advantages such as lower reflected inertia and impact forces in force control, their position control problem is more complicated than stiff actuators' one. It is shown that conventional position control systems suffer from low performance and robust position control systems excite 


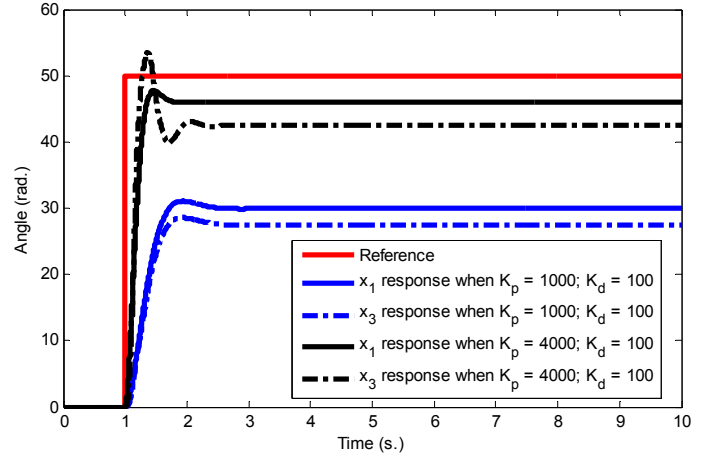

(a) Convetional position control.

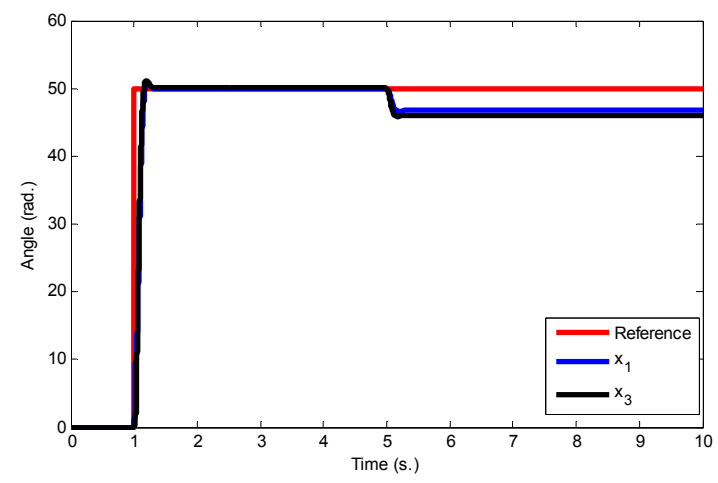

(c) Resonance ratio control.

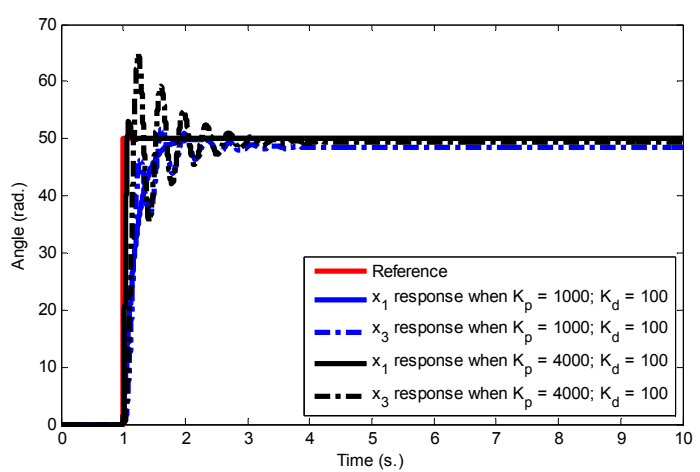

(b) Conventional acceleration based robust control.
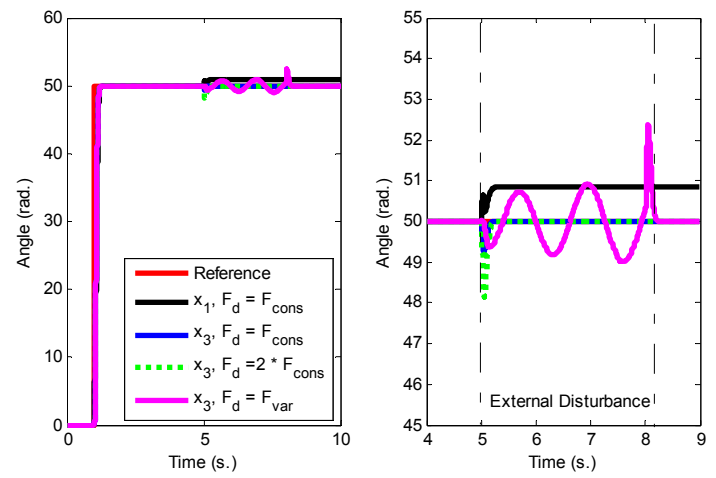

(d) Resonance ratio control and Arm DOb.

Fig.6. Position control responses of the novel SEA.

vibration at arm side. The vibration can be suppressed by decreasing the robustness, i.e., using RRC; however, the control system becomes more sensitive to external disturbances such as load. The robustness of the position control system is improved by using an Arm DOb. Consequently, a high performance robust position control system is proposed for SEAs.

The proposed novel SEA is designed by using two different springs in series so as to simply achieve passive variable stiffness. Although the control problem becomes more complicated due to the special compliant mechanical strucutre, the fundamental limitations of the conventional SEAs can be solved / relaxed by using the proposed actuator.

\section{REFERENCES}

[1] Sciliano B.; Khatib O., "Handbook of Robotics, Chapter 7, Force Control", Springer, pp. $161-207,2008$

[2] G. Zeng, "An overview of robot force control," Robotica, vol. 15, no. 15, pp. 473-482, 1997.

[3] M. Grotjahn, B. Heimann, "Model-based Feedforward Control in Industrial Robotics", International Journal of Robotics Research, vol. 21, no. 1, pp. 45-60, 2002.

[4] M. Jinno, F. Ozaki, T. Yoshimi, K. Tatsuno, M. Takahashi, M. Kanda, Y. Tamada, S. Nagataki, "Development of a force controlled robot for grinding, chamfering and polishing", IEEE International Conference on Robotics and Automation, May. 1995.

[5] P. ChanHun, H. K. Jin, P. Dong, "Development of an industrial robot manipulator for the easy and safe human-robot cooperation," International Conference on Control Automation and Systems, 27-30 Oct. 2010

[6] E. Sariyildiz, K. Ohnishi, "On the Explicit Robust Force Control via Disturbance Observer", IEEE Transaction on Industrial Electronics, vol. 62, no. 3, pp. 1581-1589, Mar. 2015.
[7] T.S. Tadele, T. de Vries, S. Stramigioli, "The Safety of Domestic Robotics: A Survey of Various Safety-Related Publications," IEEE Robotics \& Automation Magazine, vol.21, no.3, pp.134-142, Sept. 2014

[8] G. A. Pratt, P. Willisson, C. Bolton, A. Hofman, "Late motor processing in low-impedance robots: Impedance control of serieselastic actuators," American Control Conference, pp. 3245-3251, June 30 - July 2, 2004.

[9] K. Kong, , J. Bae, M. Tomizuka, "Control of Rotary Series Elastic Actuator for Ideal Force-Mode Actuation in Human-Robot Interaction Applications," IEEE/ASME Transaction on Mechatronics, vol. 14, no. 1, pp. 105-118, Feb. 2009.

[10] H. Yu, S. Huang, G. Chen, N. Thakor, "Control design of a novel compliant actuator for rehabilitation robots", Mechatronics, vol. 23, issue 8, pp. 1072-1083, Dec. 2013.

[11] K. Yuki, T. Murakami, K. Ohnishi, "Vibration control of a 2 mass system by the resonance ratio control," Trans. Inst. Elect. Eng. Jpn., vol.113-D, no. 10, pp. 1162-1169, 1993.

[12] S. Katsura, K. Ohnishi : "Force Servoing by Flexible Manipulator Based on Resonance Ratio Control," IEEE Transactions on Industrial Electronics, vol. 54, pp. 539-547, Feb., 2007

[13] E. Sariyildiz, K. Ohnishi, "Stability and Robustness of Disturbance Observer Based Motion Control Systems", IEEE Transaction on Industrial Electronics, vol. 62, no. 1, pp. 414-422, Jan. 2015.

[14] E. Sariyildiz, K. Ohnishi, "An Adaptive Reaction Force Observer Design", IEEE Transactions on Mechatronics, Apr. 2015, vol. 20, no. 2, pp. $750-760$

[15] E. Sariyildiz, K. Ohnishi, "A Guide to Design Disturbance Observer”, ASME Trans. J. Dyn. Sys., Meas., Control, Mar. 2014, vol. 136, no. 2, pp. 1-10 (021011)

[16] E. Sariyildiz, K. Ohnishi, "Analysis the Robustness of Control Systems Based on Disturbance Observer", Internat. J. Control, Oct. 2013, vol. 86, no. 10, pp. 1733-1743 